\title{
Impact of combined oil-in-water emulsions and particulate suspensions on ceramic membrane fouling and permeability recovery
}

\author{
Mays Abdalla ${ }^{\mathrm{a}}$, Mustafa Nasser ${ }^{\mathrm{a}}$, Ahmad Kayvani Fard ${ }^{\mathrm{b}, \mathrm{c}}$, Hazim Qiblawey ${ }^{\mathrm{d}}$, Abdelbaki \\ Benamor ${ }^{\mathrm{a}}$, Simon Judd ${ }^{\mathrm{a}, \mathrm{e}}$ \\ ${ }^{a}$ Gas Processing Center, Qatar University, PO Box 2713, Doha, Qatar. \\ ${ }^{\mathrm{b}}$ Qatar Environment and Energy Research Institute, Hamad Bin Khalifa University, Qatar Foundation, PO Box \\ 5825, Doha, Qatar. \\ ${ }^{\mathrm{c}}$ Division of Sustainable Development, College of Science and Engineering, Hamad Bin Khalifa University, Qatar \\ Foundation, PO Box 5825, Doha, Qatar. \\ ${ }^{\mathrm{d}}$ Department of Chemical Engineering, Qatar University, PO Box 2713, Doha, Qatar. \\ ${ }^{\mathrm{e} C}$ ranfield University, Cranfield, Beds. MK43 0AL, UK.
}

\begin{abstract}
The application of crossflow ceramic microfiltration (CFCMF) to the removal of emulsified oil from a simple analogue of raw produced water (PW) arising from oil exploration has been studied. Outcomes relate to surfactant-stabilised oil-in-water $(\mathrm{o} / \mathrm{w})$ emulsions both as a discrete emulsion and in combination with a colloidal suspension of particulate solids (bentonite). The impact on both fouling during the filtration cycle and residual fouling of the $\mathrm{ZrO}_{2}-\mathrm{TiO}_{2}$ membrane, following aggressive caustic-acid chemical chemical cleaning applied between six sequential 30-minute filtration runs, was investigated.
\end{abstract}

Results showed the addition of suspended solids to the $\mathrm{o} / \mathrm{w}$ emulsion to be extremely deleterious to sustaining both the permeability and selectivity of the membrane. The addition of 1,500 mg. $\mathrm{L}^{-1}$ of bentonite to a $10 \mathrm{vol} \%$ emulsion resulted in a permeability decrease of 3.5-5 times over that recorded for the emulsion, and 8-36 times lower than that of the bentonite suspension. Oil passage through the microfiltration membrane $(0.45 \mu \mathrm{m}$ pore size $)$ was concomitantly increased six-fold.

Tests performed to assess the cleanability of the membrane demonstrated similar differences between the three feed liquids. The permanent fouling of the membrane by the combined emulsion/suspension reduced its permeability by a factor of 16 over that attained for the emulsion-fouled membrane, or 25 times less than the residual permeability of the membrane challenged with the suspended particles. Moreover, the residual permeability of the emulsion/suspension-fouled membrane was still in decline following the sixth run. The results emphasise the importance of considering possible particle-emulsion interactions in studying membrane filtration of PW analogues.

Keywords: $\quad$ ceramic microfiltration; oil-in-water emulsion; particles; fouling; cleaning

\section{Introduction}

Produced water (PW) represents the most voluminous waste generated from petroleum industry activities, it being the wastewater from oil exploration. Removal of the suspended materials (comprising the free oil and inorganic solids) through a supplementary process following the classical hydrocyclone and gas flotation unit operations is required either to meet high discharge standards or permit reuse (Jiménez et al, 2018; Nasiri et al, 2017; Rawindran et al, 2017). Reuse opportunities onshore are largely limited by the those offered locally, but often demand desalination by reverse osmosis (Osipi et al, 2018) for which rigorous removal of suspended 
and colloidal solids is required. Offshore reuse is limited to reinjection into the reservoir (Rawindran et al, 2017), for which removal of suspended matter is required for "tight" reservoir formations of low permeability reservoirs (Jiménez et al, 2018; Chen et al, 2016). The implementation of membrane technology for this application has been the subject of a number of recent review articles (Zoubeik et al, 2018; Dickhout et al, 2017; Munirasu, 2016). In particular, the performance of ceramic membrane technology for PW filtration has been subject to increased interest over the past three years (Zsirai et al, 2018, 2016; Ebrahimi et al, 2018; Atallah et al, 2017; Thibault et al, 2017; Weschenfelder, 2016, 2015ab; Guirgis et al, 2015).

Sustaining of the membrane permeability through effective cleaning remains the key challenge in almost all membrane separation processes, and is crucially important in offshore membrane separation processes where available space is limited; maximizing the net flux presents the most effective means of minimising the technology footprint. In this regard, pilot-scale studies of cross-flow ceramic microfiltration (CFMF) membranes have demonstrated the technology to sustain comparatively high fluxes and to incur a smaller footprint than either the flotation units or nutshell filters (Zsirai et al, 2018; Weschenfelder et al, 2016). There have also been a number of predominantly bench-scale studies demonstrating the consistently high permeate water quality attained (Matos et al, 2016; Santos et al, 2016; Abadi et al, 2011).

However, there have thus far been only limited studies of the effect of combining oil-in-water emulsions and particle suspensions on filterability and selectivity for microporous ceramic membranes. There has also been very little consideration of the impact of the chemical cleaning cycle on the residual membrane permeability (the flux per unit transmembrane pressure), Moreover, the challenge to the membrane imposed by highly stabilized emulsions at high oil concentrations, such as might be encountered in untreated PW streams (i.e. upstream of the hydrocyclones), has not been extensively explored.

The current work reports on the outcomes of a bench-scale study of the application of CFMF to separate water from surfactant-stabilized highly concentrated $(10 \mathrm{v} / \mathrm{v} \%)$ oil-in-water emulsions and colloidal suspensions (1500 g. $\left.\mathrm{L}^{-1}\right)$ at different transmembrane pressures (TMPs). The selected oil concentration was based on the assumption of a feed concentration of up to 3,500 mg. $\mathrm{L}^{-1}$ oil exiting the production separator (Ray and Engelhardt, 1992) and a membrane conversion of $95-96 \%$, giving a maximum concentration of around $88,000 \mathrm{mg} . \mathrm{L}^{-1}$ oil. The study encompassed an examination of the permeability of the membrane following successive filtration and cleaning cycles, so as to quantify the relative cleanability of the membrane challenged with the emulsion, particle suspension, and combined emulsion/suspension.

\section{Materials and Methods}

\subsection{Materials}

\subsubsection{Emulsions and suspensions}

Oil-in-water emulsions were prepared from $10 \%$ vol. engine diesel oil, $863 \mathrm{~kg} . \mathrm{m}^{-3}$ density, stabilized with ethylene tetrakis, $\left[-\mathrm{CH}_{2} \mathrm{~N}\left[\left(-\mathrm{CH}_{2} \mathrm{CH}_{2} \mathrm{O}-\right)\left[-\mathrm{CH}_{2} \mathrm{CH}\left(\mathrm{CH}_{3}\right) \mathrm{O}-\right] \mathrm{H}\right]_{2}\right]_{2}$ (Sigma Aldrich, Germany), a surfactant of $1020 \mathrm{~kg} \cdot \mathrm{m}^{-3}$ density, 1-7 hydrophile-lipophile balance and $0.036 \mathrm{~N} . \mathrm{m}^{-1}$ surface tension. The colloidal material used was laboratory-grade bentonite clay, $\mathrm{Na}_{0.7} \mathrm{Al}_{3.3} \mathrm{Mg}_{0.7} \mathrm{Si}_{8} \mathrm{O}_{20}(\mathrm{OH})_{4} . \mathrm{nH}_{2} \mathrm{O}$ (Sigma Aldrich Co.,Germany). The membrane cleaning chemicals, sodium hydroxide $(\mathrm{NaOH})$ and nitric acid $\left(\mathrm{HNO}_{3}\right)$, were both supplied by BDH Chemicals (UK). 


\subsubsection{Membrane and filtration unit}

A $90 \mathrm{~mm}$-diameter zirconium dioxide-titanium dioxide $\left(\mathrm{ZrO}_{2}-\mathrm{TiO}_{2}\right)$ ceramic disc filter was used in the study, with a rated pore size of $0.45 \mu \mathrm{m}$. The Spiralab gasket generates a crossflow path of $\sim 5 \mathrm{~mm}$ width and height, providing a total active membrane area of $29.1 \mathrm{~cm}^{2}$ from the nominally total available area of $56.3 \mathrm{~cm}^{2}$.

The apparatus (Fig. 1) comprised a diaphragm pump (3), rated at $1.35 \mathrm{~L}^{\mathrm{min}} \mathrm{min}^{-1}$ at 6.9 bar and supplied by Aqua Leader (Quebec, Canada), connected to a $3 \mathrm{~L}$ feed reservoir (1). A liquid filter (2) was installed inside the feed tank to ensure a homogenous medium and stabilize the emulsions during operation. The pipework was fitted with valves for feed bypass (6) and retentate throttling (8), as well as a feed pressure gauge (6). Filtrate from the ceramic membrane crossflow disc holder (Spirlab, SterliTech, US), of stainless steel construction, was collected in the permeate reservoir (10) and the rejected (retentate) stream directed to drain. All pipes and fittings were based on $6 \mathrm{~mm}$ diameter pipework.

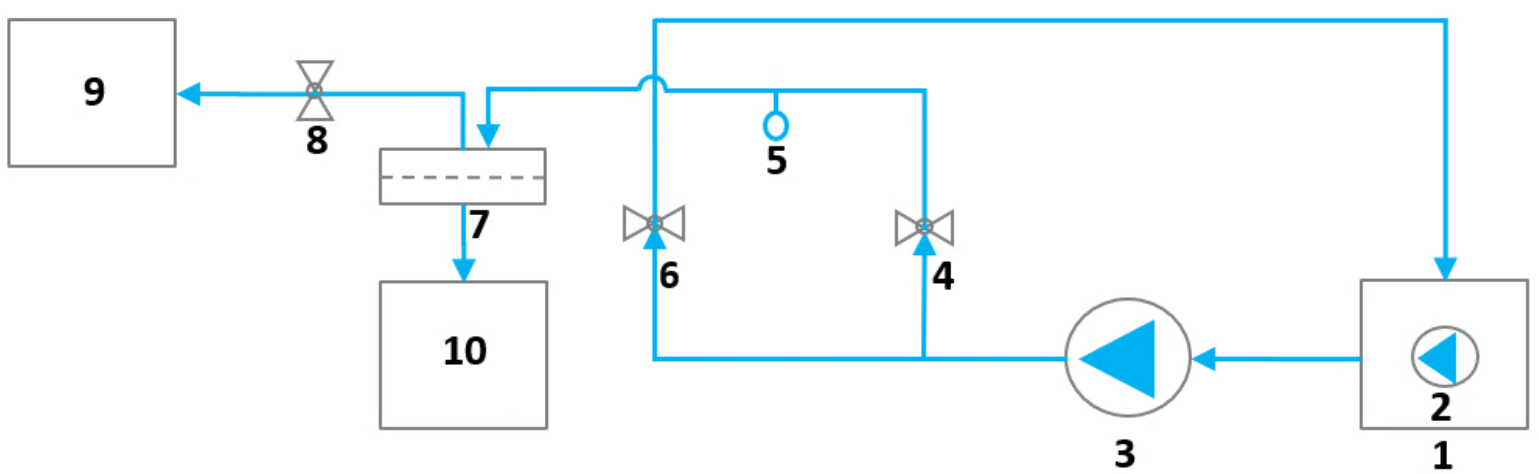

Figure 1: Schematic of the experimental setup. See accompanying text for numbering.

\subsection{Methods}

\subsubsection{Analytical instrumentation}

The emulsion interfacial tension was determined by the pendant-drop method (Drop Shape Analyzer DSA100, KRÜSS GmbH, Germany) according to standard methods (APHA, 2012) at surfactant concentrations of $0.25-3 \mathrm{vol} \%$. Zeta potential was measured of using a Zetasizer ZEN3600 (Malvern Instruments Ltd., UK) at room temperature for samples diluted 100x in DI water, the instrument set to iterate at least 12 times. Feed and permeate oil concentration was determined using Total Organic Carbon Analyzer TOC-L (Shimadzu, Japan). Permeate turbidity was recorded using a Hach $2100 \mathrm{~N}$ bench top turbidity meter (Hach, US).

It was assumed that the trend in percentage oil rejection is reasonably well represented by that of TOC, as implied by previously reported rejection measurements of COD and oil concentration on single samples of oil refinery wastewater (Santos et al, 2016). Since the measured TOC rejection values are slightly slightly lower than those for oil rejection the data trends provided are likely to be more conservative than those for oil rejection specifically.

\subsubsection{Sample preparation and experiments}

Oil/water emulsions (Table 1) were prepared by blending the set concentration of surfactant (between $0.5 \%$ vol. up to $5 \%$ vol.) with the oil $\left(10 \%\right.$ by volume, $\left.86,300 \mathrm{mg} . \mathrm{L}^{-1}\right)$ in DI water at a homogeniser speed of $3500 \mathrm{rpm}$ for 10-15 minutes, the oil being added incrementally via a burette. The optimum surfactant concentration for providing stable emulsions was inferred from 
monitoring the phase separation with time over a 30 minute period (Section 3.1.1). The same preparation procedure was carried out for generating oil-in-water emulsions with colloidal suspensions, blending the oil with a $1.5 \mathrm{~g} . \mathrm{L}^{-1}$ bentonite suspension.

Table 1: Droplet and particle characteristics, full stabilised

\begin{tabular}{|c|c|}
\hline Parameter & Value \\
\hline Oil $^{1}$ concentration, mg.L $\mathrm{L}^{-1}(\mathrm{v} / \mathrm{v} \%)$ & $86,300(10 \%)$ \\
\hline Oil $^{1}$ droplet size $\left(d_{50}\right), \mu \mathrm{m}$ & $4.6^{3}$ \\
\hline Oil $^{1}$ density, kg.m ${ }^{-3}$ & 863 \\
\hline Particle $^{2}$ concentration, g. $\mathrm{L}^{-1}$ & 1,500 \\
\hline $\operatorname{Particle}^{2}$ size $\left(d_{50}\right), \mu \mathrm{m}$ & 9.7 \\
\hline Particle $^{2}$ density, $\mathrm{kg} \cdot \mathrm{m}^{-3}$ & 2,460 \\
\hline Temperature range, ${ }^{\circ} \mathrm{C}$ & $24-26$ \\
\hline
\end{tabular}

The $\mathrm{pH}$ was maintained at $9.0 \pm 0.2$ throughout the study: scoping trials revealed the prepared suspensions to be substantially stable at this $\mathrm{pH}$, with no apparent phase separation of the feed solutions over the course of the tests.

30-minute filtration tests were conducted at TMPs ranging from 0.2 bar to 1.4 bar, the permeate flux being recorded and samples collected every minute for the first 10 minutes and then at 10 minutes intervals subsequently. A maximum run time of 30 minutes was used, it being considered that this was a sufficient time period to allow the initial rapid flux decline to be defined (Zsirai et al, 2016). The equilibrium flux was not attained within this time period, as is the case for most studies conducted in this area.

At the rated pump flow rate the crossflow velocity (CFV) in the retentate channel was around $0.9 \mathrm{~m} . \mathrm{s}^{-1}$ and, at the highest flux recorded in the study $\left(\sim 2000 \mathrm{~L} \cdot \mathrm{m}^{-2} \cdot \mathrm{h}^{-1}\right.$ or LMH), the conversion below $8 \%$. Impacts of conversion on the CFV were thus considered negligible, and the retentate channel $\mathrm{CFV}$ assumed to be constant at $0.9 \mathrm{~m} \cdot \mathrm{s}^{-1}$ throughout.

\subsubsection{Membrane cleaning}

Membrane chemical cleaning was based on the protocol of Zsirai et al (2016), employing the same protocol (Table 2) after every run. The membrane was initially flushed through with DI water in situ and then submerged in a 20 g. $\mathrm{L}^{-1}$ sodium hydroxide solution for 30 minutes at $80^{\circ} \mathrm{C}$. Following rinsing with DI water, a similar soak at the same temperature and duration in 5 g. $\mathrm{L}^{-1}$ nitric acid solution was applied prior to rinsing twice with deionised water. Following suxch cleaning the flux:pressure trend for a clean water feed was generated to obtain the clean water permeability $\left(K_{w}\right)$ in the slope.

Table 2: Cleaning-in-place procedure

\begin{tabular}{l|l}
\hline $\begin{array}{l}\text { Reagent, } \sim 50 \mathrm{~L} \\
\text { aliquots }\end{array}$ & Soak time and temperature \\
\hline 1. Mains water & $30 \mathrm{~min}$ at $22-24^{\circ} \mathrm{C}$ \\
2. $2 \% \mathrm{NaOH}$ & $30 \mathrm{~min}$ at $\mathrm{pH} 13$ and $80^{\circ} \mathrm{C}$ \\
3. Mains water & $30 \mathrm{~min}$ at $22-24^{\circ} \mathrm{C}$ \\
4. $0.5 \%$ nitric acid & $30 \mathrm{~min}$ at $\mathrm{pH} 2$ and $80^{\circ} \mathrm{C}$ \\
5. Mains water & $30 \mathrm{~min}$ at $22-24^{\circ} \mathrm{C}$ \\
\hline
\end{tabular}




\section{Results and discussion}

\subsection{Data quality and reproducibility}

Fluctuations in both the flux and the TOC breakthrough trends at in the initial stages of the tests were attributable to challenges in manually controlling the retentate flow to sustain the crossflow velocity and transmembrane pressure. The initial period of operation represented that during which the rate of change of flux, and the associated fouling rate, was at its greatest.

The phase separation data represent the average of three tests. For these triplicates, $95 \%$ of the standard deviation (SD) values were less than 5\%, and the average overall SD value was $2.2 \%$. The zeta potential measurements were also triplicated, and in this case $92 \%$ of the standard deviation (SD) values for the triplicates were less than $5 \%$, with an average overall SD value of $2.8 \%$. The flux decline measurements were duplicated. The deviation between the duplicates ranged from less than $1 \%$ at the highest TMP values to generally between 2 and $5 \%$ at the lowest values.

\subsection{Oil-in-water emulsion characterization}

The expected trend of suppressed phase separation with increased surfactant concentration was demonstrated at surfactant concentrations above $0.5 \%$ (Fig. 2). In the absence of surfactant almost $15 \%$ of the oil separated in less than one minute, with only a slight improvement in stability at surfactant concentrations up to $0.75 \%$. Stabilization improved significantly at a surfactant concentration of $1 \%$, the emulsion remaining stable for 2.5 minutes. At concentrations of $2 \%$ and above the emulsion was nominally stabilized, or "tight", with no phase separation evident over a 30-minute period.

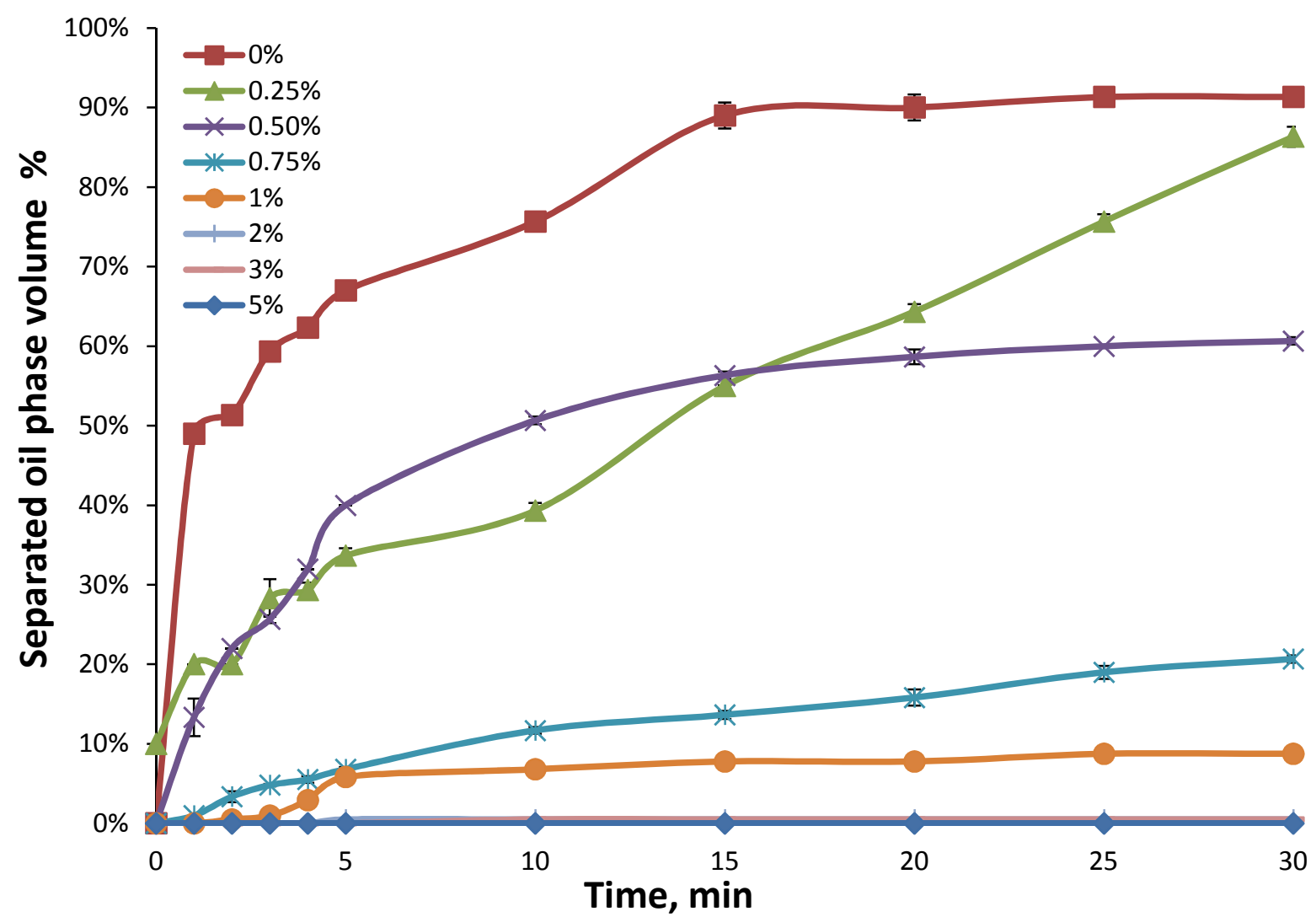

Figure 2: Effect of vol $/ \mathrm{vol} \%$ surfactant concentration $(0-5 \%)$ on $\mathrm{o} / \mathrm{w}$ emulsion stability, 86,300 mg.L $L^{-1}$ oil 
Correlation of surfactant concentration with zeta potential, $\zeta$, revealed $\zeta$ to decrease from -11.8 $\mathrm{mV}$ at $0.25 \%$ concentration to $-20.1 \mathrm{mV}$ at $0.75 \%$ before stabilizing at $-16 \mathrm{mV}$ at concentrations above $1 \%$ (Fig. 3). This trend is similar to that reported previously for diesel oil in brine solution stabilized by xanthan gum and aluminium stearate (Jha et al., 2016). The interfacial tension (IFT) trend indicated a dramatic decrease in IFT from 16 to 4.6 surfactant concentration between 0 and $0.125 \%$ surfactant concentration (Fig. 3), with a shallower decline thereafter to $0.99 \mathrm{mN} . \mathrm{m}^{-1}$ at concentrations of $2 \%$ or more.

A surfactant concentration of $2 \%$ was thus selected for all subsequent membrane filtration tests. At this concentration the emulsion characteristics, and specifically the droplet size, were as indicated in Table 1.

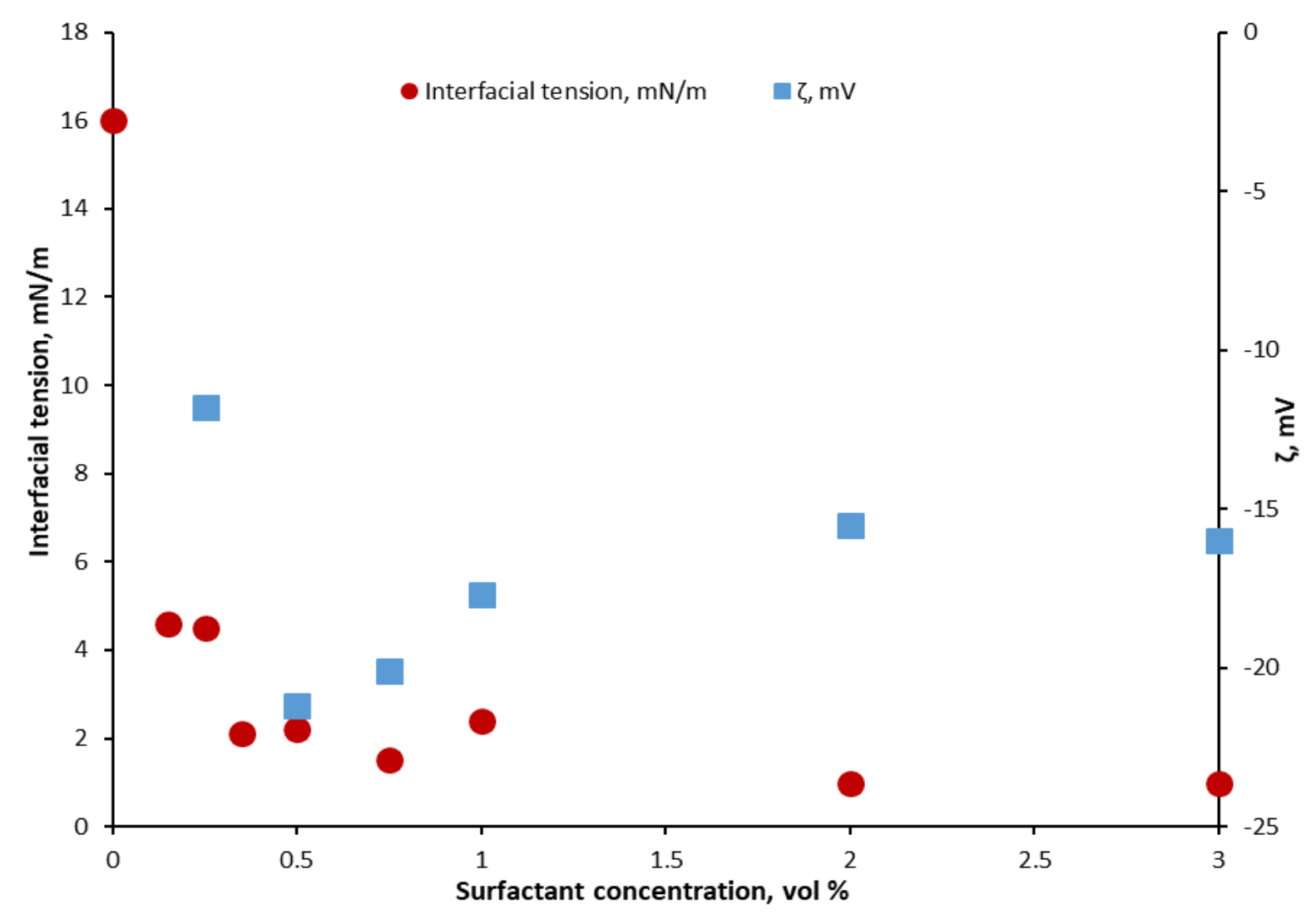

Figure 3: Effect of surfactant concentration on interfacial tension and zeta potential

\subsection{Membrane filtration, single run-tests}

\subsubsection{Flux decline}

Permeate flux for the emulsion demonstrated the expected trends of increasing with pressure and decreasing with time, though the transient trends were not consistent (Fig. 4) with the decline at 0.7 bar being steeper than for all other TMPs. This possibly reflects the lack of control provided by the relatively low CFV and the associated fouling. Flux values after 30 minutes increased from $41 \mathrm{LMH}$ at the lowest TMP of 0.2 bar to 346 at 1.4 bar (with corresponding permeabilities of 205 and $247 \mathrm{LMH} . b a r^{-1}$ ), from initial fluxes of 98 and $475 \mathrm{LMH}$ respectively. This compares with fluxes of 194-240 LMH and steady-state permeabilities (after $24 \mathrm{~h}$ ) of 97120 LMH.bar $^{-1}$ reported by Weschenfelder et al (2015b) for a similar CFMF membrane challenged with a PW analogue and 295-312 LMH and corresponding permeabilities of 118125 LMH.bar ${ }^{-1}$ for an alumina membrane challenged with real PW (Reyhani and Mashhadi 
Meighani, 2015). These studies refer to CFVs of 2-3 m.s $\mathrm{s}^{-1}$, somewhat higher than the value for the current study. The higher permeabilities recorded in the current study most probably reflect the conditions not having reached steady state after 30 minutes.

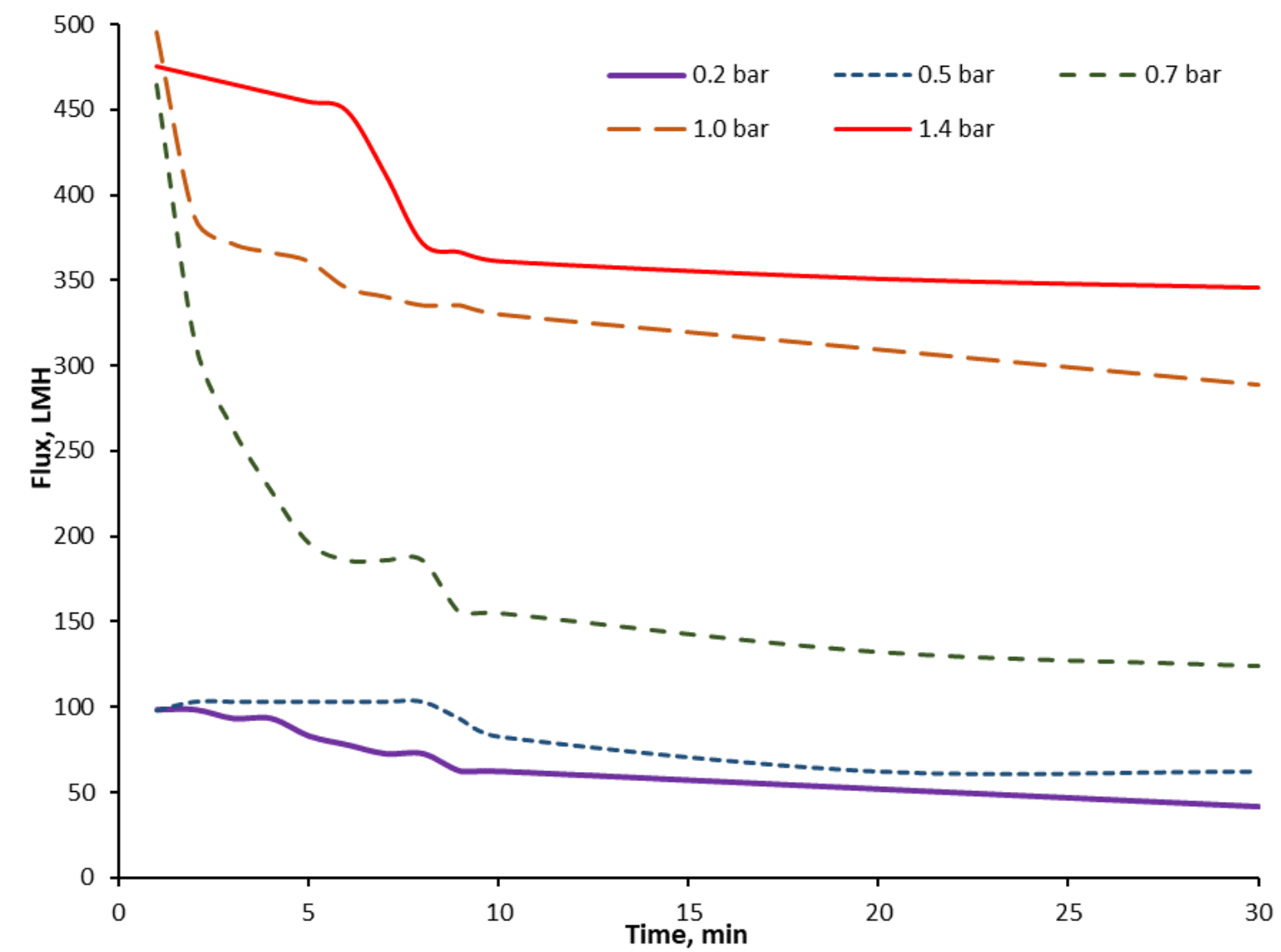

Figure 4: Permeate flux transients vs TMP for stabilised o/w emulsion, oil concentration and droplet size given in Table 1.

For the suspension the flux decline was, as expected, shallower and the flux after 30 minutes of filtration $\left(J_{30}\right)$ correspondingly higher (generally between 555 and $675 \mathrm{LMH}$ across the range of TMPs applied) than for the emulsion. The fouling layer from a granular, non-deformable particle greater in size than that of the membrane pore would be expected to incur a much smaller reduction in permeability that a deformable, hydrophobic oil droplet.

The combination of emulsified oil and suspended solids resulted in a significantly reduced permeability, as reflected in the $J_{30}$ :TMP correlations (Fig. 5). The permeability for the combined liquid was 3.5-5 times lower that of the emulsified oil and 8-36 times lower than the suspension, the difference increasing with decreasing TMP. This demonstrates that under the conditions employed in the trials the particulate matter in the suspension does not form a protective later on the membrane surface but instead exacerbates the fouling propensity of the emulsified oil. 


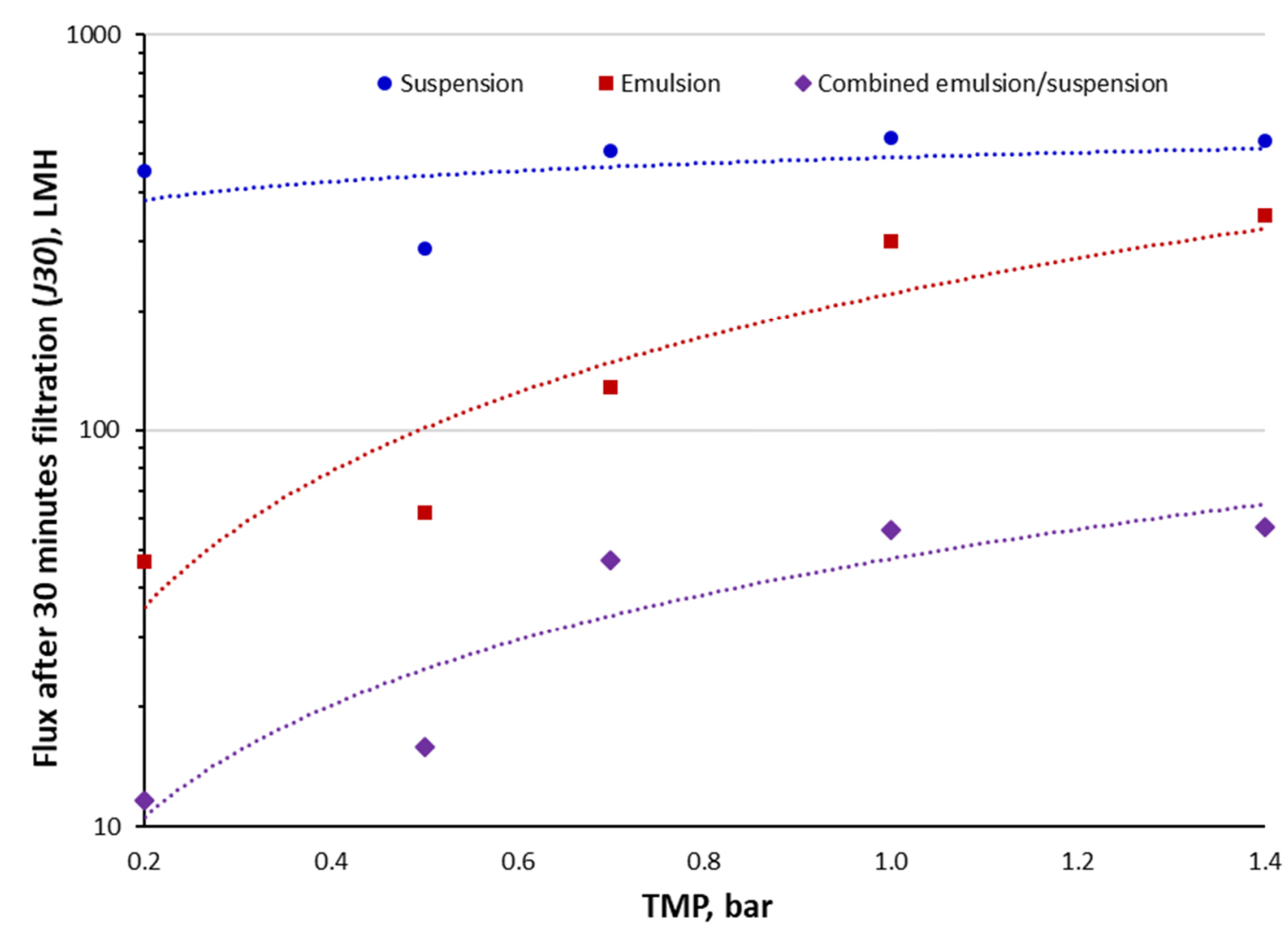

Figure 5: Permeate flux after 30 minutes of filtration $\left(J_{30}\right)$ vs. TMP for the three liquid feeds

\subsubsection{Permeate quality}

For the emulsion, permeate TOC concentrations ranged from 10,000 to $24,000 \mathrm{mg} . \mathrm{L}^{-1}$, corresponding to rejections of $91 \%$ to $79 \%$, across all TMPs applied. No trend was evident either with time or TMP (Fig. 6), and at 30 minutes observed differences in rejection with TMP were negligible. TOC removal values reported in other studies have varied significantly according to the distribution of the oil between the free and dissolved phase, and have tended to report small decreases in rejection with TMP both for surfactant-stabilised emulsions (Zhu et al, 2017; Matos et al, 2016; Abassi et al, 2012) and real wastewater (Abadi et al, 2011). These previous studies have reported rejection values well in excess of $90 \%$. The lower rejections recorded in the current study reflect the relatively large pore size of the membrane used $(0.45$ $\mu \mathrm{m}$ ), compared with membrane pore sizes of $0.1 \mu \mathrm{m}$ or less employed by previous researchers, but with no apparent pressure-related breakthrough of the oil droplets. According to the computations of Zhu et al (2017) breakthrough of the droplets would appear to be unlikely under the conditions of the test $(<1.4$ bar TMP, $0.45 \mu \mathrm{m}$ maximum pore size, $4.6 \mu \mathrm{m}$ median droplet size).

As expected, suspended solids were effectively rejected, by more than $99.6 \%$, for both the suspension, with just a slightly reduced rejection at the highest TMP $(99.6 \%$ cf. $99.8 \%$ on average for all other TMP values). 


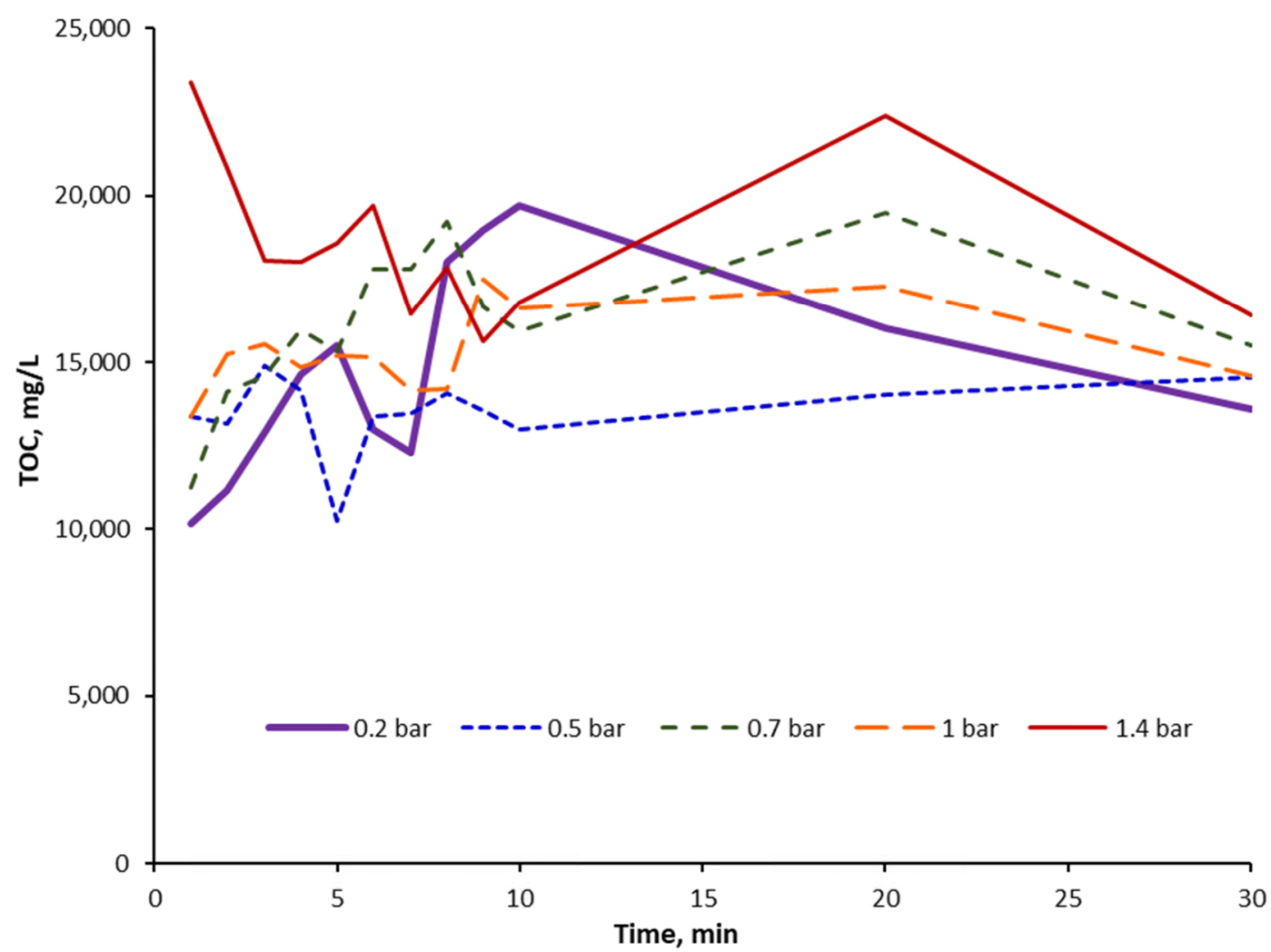

Figure 6: Permeate TOC vs. TMP for $\mathrm{o} / \mathrm{w}$ emulsion, oil concentration and droplet size given in Table 1.

The combined emulsion/suspension, despite generating an apparently less permeable fouling layer (Section 3.2.1), nonetheless resulted in a greater permeation of the oil through the membrane leading to TOC concentrations almost six times greater than those for the $\mathrm{o} / \mathrm{w}$ emulsion (Fig. 7). Moreover, the turbidity of the permeate was also affected at the two highest TMPs applied, reducing TSS rejection to between 95 and $97 \%$ (from 99.6-99.8\% for the suspension alone). This implies that, at these two TMPs, breakthrough of suspended material (presumably free oil) is around 20 times greater for the combined emulsion/suspension than for the suspension alone.

The impact of the addition of suspended material to a concentrated stabilized emulsion was thus highly deleterious to the selectivity, as well as to permeability, of the microporous membrane. This outcome is contrary to at least one previous report (Panpanit and Visvanathan, 2001) demonstrating improved performance from the addition of bentonite to an oily-water emulsion by a polymeric membrane, though the conditions employed significantly differed to those of the current study. Since all physical conditions relating to the flow were the same as for the emulsion, it must be assumed that breakthrough relates to synergistic interactions between the oil, particles and membrane. 


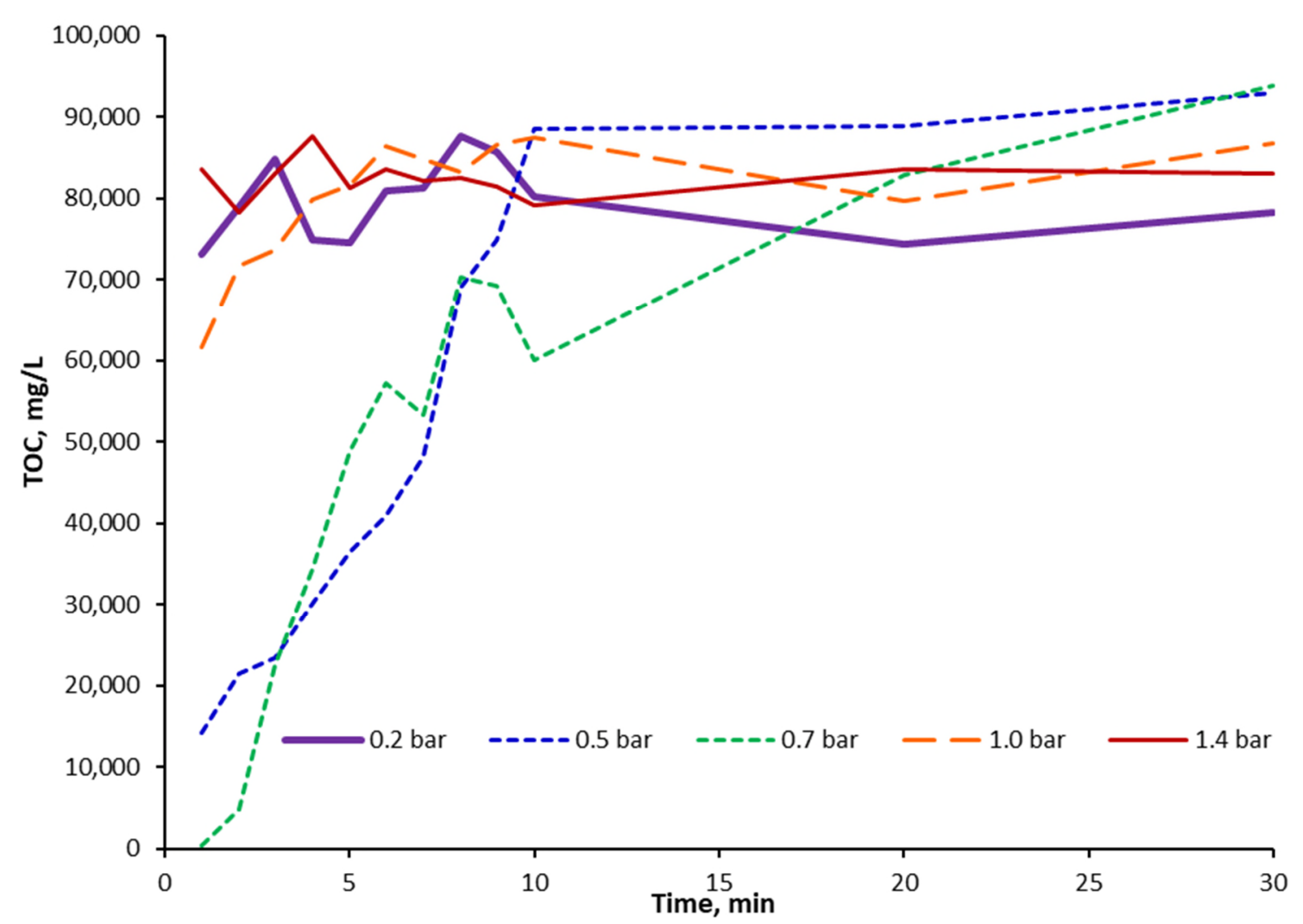

Figure 7: Permeate TOC vs. TMP for combined emulsion/suspension

\subsection{Sequential runs}

The efficacy of chemical cleaning was studied by undertaking sequential runs under the same operating conditions following chemical cleaning using the same protocol between each run, and measuring the pure water permeability following each chemical clean. Results for the stabilized o/w emulsion (Fig. 8) indicate that a discontinuous reduction in residual clean-water permeability, i.e. the gradient of the trends displayed in Figure 8, takes place - specifically between Runs 1 and 2 and Runs 3 and 4. Compared to a clean water permeability of 1185 LMH.bar ${ }^{-1}$ for the original material, the permeability decreased to 582 LMH.bar $^{-1}$ following Run 1 and then from 347 to $321 \mathrm{LMH}^{\mathrm{L}} \mathrm{bar}^{-1}$ between Runs 4 and 6. The residual permeability was thus still in decline after the sixth run, notwithstanding the frequent and rigorous chemical clean.

A comparison of the clean water permeability $\left(K_{w}\right)$ values, pertaining to residual membrane fouling, for the $\mathrm{o} / \mathrm{w}$ emulsion, suspension, and the combined emulsion/suspension mixture indicates significant differences in behaviour across the three liquid media (Fig. 9). Whereas the emulsion and suspension residual $K_{w}$ values had both almost stabilized after Run 6 , to values of around 350 and 550 LMH.bar ${ }^{-1}$ respectively, the corresponding value for the combined liquid feed was both very significantly lower, at only 22 LMH.bar $^{-1}$, and still declining after Run 6 . This arises despite the organic and solids load received by the more highly-fouled membrane being much lower over the course of the 30-minute run, commensurate with the lower flux sustained. 


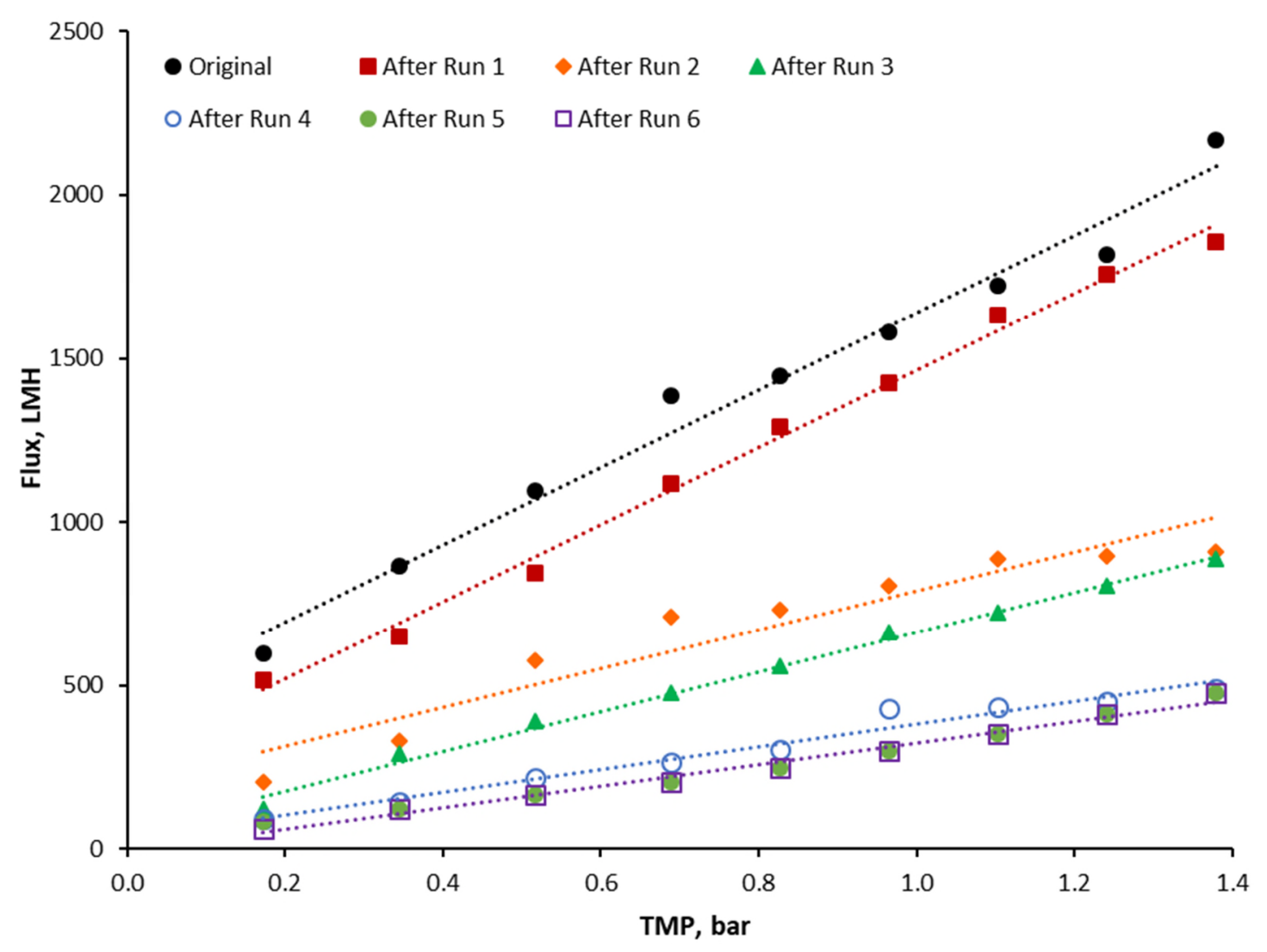

Figure 8: Clean water flux vs. TMP following sequential 30-minute runs, o/w emulsion

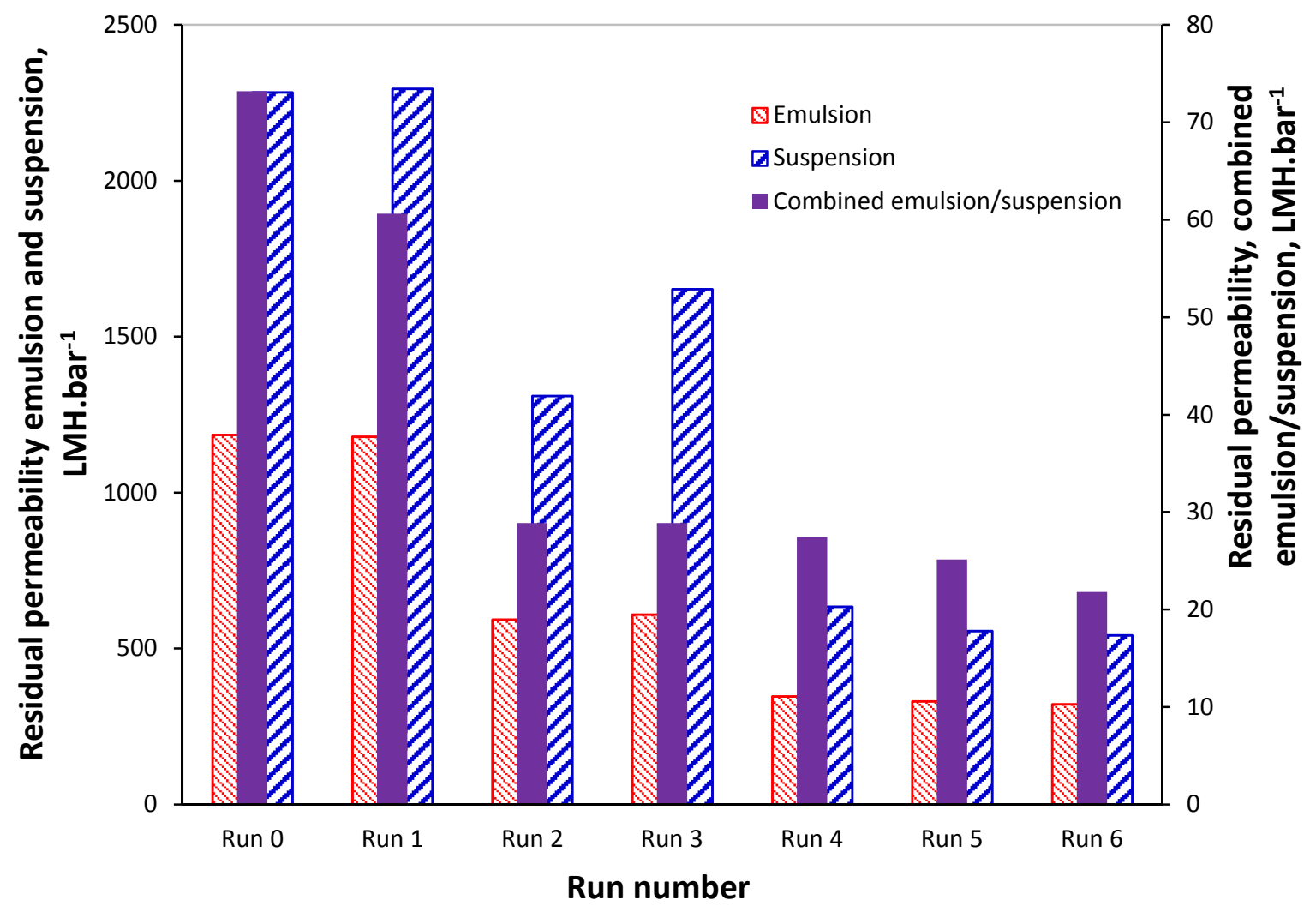

Figure 9: Residual permeability vs. run number for all three liquid media 
A number of published bench-scale studies have demonstrated significant differences in membrane fouling propensity between synthetic and real wastewaters (Madaeni et al., 2013; Ebrahimi et al, 2010; Chakrabarty et al, 2010). These differences are often attributed characteristics of the oil and solution, such as the droplet size distribution, conductivity and $\mathrm{pH}$ (Dickhout et al, 2017; Zhu et al, 2017; Chakrabarty et al., 2010). However, such phenomena cannot account for the behavioural differences observed in the current study, where the only difference between the emulsion and the combined emulsion/suspension is the addition of the particles. Moreover, the persistence of permanent fouling, i.e. fouling not removed by chemical cleaning, when the particles are present appears to have gone unreported before. On the other hand, it may at least in part account for the low permeabilities reported both for both the above bench-scale trials and for pilot studies (Guirgis et al, 2015; Prado-Rubio et al, 2012; Pedenaud et al, 2011) performed on real PW streams.

\section{Conclusion}

The fouling trends of a $0.45 \mu \mathrm{m}$ pore size crossflow ceramic microfiltration (CFMF) membrane filtering a concentrated produced water (PW) analogue have been studied. The membrane, operating in crossflow mode, was challenged with a surfactant-stabilised oil-in-water $(\mathrm{o} / \mathrm{w})$ emulsion and a colloidal particle suspension, both individually and in combination. Impacts were recorded for (a) flux decline during a single filtration cycle, (b) permeate water quality with reference to TOC and turbidity, and (c) residual (permanent) fouling following a fixed chemical clean applied between sequential filtration tests.

The addition of the particulate solids (at $\left.1,500 \mathrm{mg} . \mathrm{L}^{-1}\right)$ to the stabilised emulsion $(10 \% \mathrm{v} / \mathrm{v})$ was found to reduce the membrane permeability over the course of the filtration test by 3.5-5 times compared with the emulsion alone, and a 8-36 times reduction compared with the suspension. This was accompanied by a six-fold factorial increase in the TOC passage through the membrane.

The trend in permanent fouling, as manifested in the membrane permeability following chemical cleaning, was similar across the three liquid matrices. Based on six short runs with a standard chemical clean between each, the residual permeability of the membrane challenged with the combined emulsion/suspension was 16 -fold lower than for fouling by the emulsion alone, and 25 times less than for suspended particles fouling.

Whilst the absolute output values recorded for the study are unlikely to be representative, given the small scale of operation, the low temperatures employed and the use of analogue material to represent the emulsified and suspended contaminants, the observed impact of the suspended solids on the membrane permeability and permeate quality is likely to be generally valid. The results indicate that suspended inorganic particles may significantly deleteriously impact on the microfiltration of emulsified oil. This is contrary to the intuitive assumption of a measure protection of the membrane from fouling afforded by the formation of a cake layer by the granular solids, and suggests that care must be taken in selecting appropriate analogues to represent PW when conducting membrane filtration studies. 


\section{Acknowledgements}

The kind assistance of the technical staff at the Gas Processing Center at Qatar University is gratefully acknowledged.

\section{References}

Abadi, S. R. H., Sebzari, M. R., Hemati, M., Rekabdar, F., \& Mohammadi, T. (2011). Ceramic membrane performance in microfiltration of oily wastewater. Desalination, 265(1-3), 222-228.

Abadi, S. R. H., Sebzari, M. R., Hemati, M., Rekabdar, F., \& Mohammadi, T. (2011).

Ceramic membrane performance in microfiltration of oily wastewater. Desalination, 265(1-3), 222-228.

Abbasi, M., Salahi, A., Mirfendereski, M., Mohammadi, T., Rekabdar, F., \& Hemmati, M. (2012). Oily wastewater treatment using mullite ceramic membrane. Desal. Water Treat., 37(13), 21-30

Abbasi, M., Salahi, A., Mirfendereski, M., Mohammadi, T., Rekabdar, F., \& Hemmati, M. (2012). Oily wastewater treatment using mullite ceramic membrane. Desal. Water Treat., $37(1-3), 21-30$

American Public Health Association (APHA). American Water Works Association (AWWA) and Water Environment Federation (WEF): Standard Methods for the Examination of Water and Wastewater, 22nd Edition (2012).

American Public Health Association (APHA). American Water Works Association (AWWA) and Water Environment Federation (WEF): Standard Methods for the Examination of Water and Wastewater, 22nd Edition (2012).

Atallah, C., Tremblay, A. Y., and Mortazavi, S. (2017). Silane surface modified ceramic membranes for the treatment and recycling of SAGD produced water. J. Petrol. Sci. Eng., 157, 349-358.

Atallah, C., Tremblay, A. Y., and Mortazavi, S. (2017). Silane surface modified ceramic membranes for the treatment and recycling of SAGD produced water. J. Petrol. Sci. Eng., $157,349-358$.

Chakrabarty, B., Ghoshal, A. K., and Purkait, M. K. (2010). Cross-flow ultrafiltration of stable oil-in-water emulsion using polysulfone membranes. Chem. Eng. J., 165(2), 447-456.

Chakrabarty, B., Ghoshal, A. K., and Purkait, M. K. (2010). Cross-flow ultrafiltration of stable oil-in-water emulsion using polysulfone membranes. Chem. Eng. J., 165(2), 447-456.

Chen, P., Willingham, T., Morrow, T. I., Al Sowaidi, A. K., Stojkovic, D., Ning, J., and Gao, B. (2017). Assessment of produced water reinjection in a giant carbonate reservoir. Paper presented at the Society of Petroleum Engineers - SPE Abu Dhabi International Petroleum Exhibition and Conference, Jan 2017.

Chen, P., Willingham, T., Morrow, T. I., Al Sowaidi, A. K., Stojkovic, D., Ning, J., and Gao, B. (2017). Assessment of produced water reinjection in a giant carbonate reservoir. Paper presented at the Society of Petroleum Engineers - SPE Abu Dhabi International Petroleum Exhibition and Conference, Jan 2017.

Dickhout, J. M., Moreno, J., Biesheuvel, P. M., Boels, L., Lammertink, R. G. H., and de Vos, W. M. (2017). Produced water treatment by membranes: A review from a colloidal perspective. J. Coll. Interface Sci., 487, 523-534. 
Dickhout, J. M., Moreno, J., Biesheuvel, P. M., Boels, L., Lammertink, R. G. H., and de Vos, W. M. (2017). Produced water treatment by membranes: A review from a colloidal perspective. J. Coll. Interface Sci., 487, 523-534.

Ebrahimi, M., Kerker, S., Schmitz, O., Schmidt, A. A., and Czermak, P. (2018). Evaluation of the fouling potential of ceramic membrane configurations designed for the treatment of oilfield produced water. Sep. Sci. Technol., 53(2), 349-363.

Ebrahimi, M., Kerker, S., Schmitz, O., Schmidt, A. A., and Czermak, P. (2018). Evaluation of the fouling potential of ceramic membrane configurations designed for the treatment of oilfield produced water. Sep. Sci. Technol., 53(2), 349-363.

Ebrahimi, M., Willershausen, D., Ashaghi, K.S., Engel, L., Placido, L., Mund, P., Bolduan, P., and Czermak (2010). Investigations on the use of different ceramic membranes for efficient oil-field produced water treatment. Desalination, 250, 991-6.

Ebrahimi, M., Willershausen, D., Ashaghi, K.S., Engel, L., Placido, L., Mund, P., Bolduan, P., and Czermak (2010). Investigations on the use of different ceramic membranes for efficient oil-field produced water treatment. Desalination, 250, 991-6.

Guirgis, A., Gay-de-Montella, R., and Faiz, R. (2015). Treatment of produced water streams in SAGD processes using tubular ceramic membranes. Desalination, 358, 27-32.

Guirgis, A., Gay-de-Montella, R., and Faiz, R. (2015). Treatment of produced water streams in SAGD processes using tubular ceramic membranes. Desalination, 358, 27-32.

Jha, P., V. Mahto, and V. Saxena (2016), Study the effects of xanthan gum and aluminium stearate on the properties of oil-in-water emulsion drilling fluids. Arab. J. Sci. Eng. 41(1) 143153.

Jha, P., V. Mahto, and V. Saxena (2016), Study the effects of xanthan gum and aluminium stearate on the properties of oil-in-water emulsion drilling fluids. Arab. J. Sci. Eng. 41(1) 143153.

Jiménez, S., Micó, M. M., Arnaldos, M., Medina, F., and Contreras, S. (2018). State of the art of produced water treatment. Chemosphere, 192, 186-208.

Jiménez, S., Micó, M. M., Arnaldos, M., Medina, F., and Contreras, S. (2018). State of the art of produced water treatment. Chemosphere, 192, 186-208.

Madaeni, S, Gheshlaghi, A. and Rekabdar F. (2013). Membrane treatment of oily wastewater from refinery processes, Asia-Pac. J. Chem. Eng., 8(1), 45-53.

Madaeni, S, Gheshlaghi, A. and Rekabdar F. (2013). Membrane treatment of oily wastewater from refinery processes, Asia-Pac. J. Chem. Eng., 8(1), 45-53.

Matos, M., Gutiérrez, G., Lobo, A., Coca, J., Pazos, C., and Benito, J. M. (2016). Surfactant effect on the ultrafiltration of oil-in-water emulsions using ceramic membranes. J. Membr. Sci., 520, 749-759.

Matos, M., Gutiérrez, G., Lobo, A., Coca, J., Pazos, C., and Benito, J. M. (2016). Surfactant effect on the ultrafiltration of oil-in-water emulsions using ceramic membranes. J. Membr. Sci., 520, 749-759.

Munirasu, S., Haija, M. A., \& Banat, F. (2016). Use of membrane technology for oil field and refinery produced water treatment - A review. Proc. Safety Env. Protect. 100, 183-202.

Munirasu, S., Haija, M. A., \& Banat, F. (2016). Use of membrane technology for oil field and refinery produced water treatment - A review. Proc. Safety Env. Protect. 100, 183-202. 
Nasiri, M., Jafari, I., and Parniankhoy, B. (2017). Oil and Gas Produced Water Management: A Review of Treatment Technologies, Challenges, and Opportunities, Chem. Eng. Comm. 204(8), 990-1005.

Nasiri, M., Jafari, I., and Parniankhoy, B. (2017). Oil and Gas Produced Water Management: A Review of Treatment Technologies, Challenges, and Opportunities, Chem. Eng. Comm. 204(8), 990-1005.

Osipi, S. R., Secchi, A. R., and Borges, C. P. (2018). Cost assessment and retro-technoeconomic analysis of desalination technologies in onshore produced water treatment. Desalination, 430, 107-119.

Osipi, S. R., Secchi, A. R., and Borges, C. P. (2018). Cost assessment and retrotechnoeconomic analysis of desalination technologies in onshore produced water treatment. Desalination, 430, 107-119.

Panpanit, S., and Visvanathan, C. (2001). The role of bentonite addition in UF flux enhancement mechanisms for oil/water emulsion. J. Membr. Sci., 184(1), 59-68.

Panpanit, S., and Visvanathan, C. (2001). The role of bentonite addition in UF flux enhancement mechanisms for oil/water emulsion. J. Membr. Sci., 184(1), 59-68.

Pedenaud, P., Heng, S., Evans, W., and Bieonneau, D. (2011). Ceramic membrane and core pilot results for produced water, OTC-22371-PP, paper presented at Offshore Technology Conference, 4-6 Oct, Rio de Janeiro.

Pedenaud, P., Heng, S., Evans, W., and Bieonneau, D. (2011). Ceramic membrane and core pilot results for produced water, OTC-22371-PP, paper presented at Offshore Technology Conference, 4-6 Oct, Rio de Janeiro.

Prado- Rubio, O.A., Cardona, D., Svendsen, T., and Yuan L. (2012). SiC membrane pilot plant ultrafiltration test for produced water treatment, Ocelote field - Hocol (COLOMBIA). Company report, Liqtech.

Prado- Rubio, O.A., Cardona, D., Svendsen, T., and Yuan L. (2012). SiC membrane pilot plant ultrafiltration test for produced water treatment, Ocelote field - Hocol (COLOMBIA). Company report, Liqtech.

Rawindran, H., Krishnan, S., and Sinnathambi, C. M. (2017). A review on overboard CEOR discharged produced water treatment and remediation. Paper presented at the IOP Conference Series: Materials Science and Engineering, 206(1).

Rawindran, H., Krishnan, S., and Sinnathambi, C. M. (2017). A review on overboard CEOR discharged produced water treatment and remediation. Paper presented at the IOP Conference Series: Materials Science and Engineering, 206(1).

Ray, J.P., and Engelhardt, F.R., (Eds.). Produced Water: Technological/Environmental Issues and Solutions, Springer, NY, 1992.

Reyhani, A., and Mashhadi Meighani, H. (2016). Optimal operating conditions of micro- and ultra-filtration systems for produced-water purification: Taguchi method and economic investigation. Desal. Water Treat. 57(42) 19642-19654.

Reyhani, A., and Mashhadi Meighani, H. (2016). Optimal operating conditions of micro- and ultra-filtration systems for produced-water purification: Taguchi method and economic investigation. Desal. Water Treat. 57(42) 19642-19654. 
Santos, B., Crespo, J. G., Santos, M. A., and Velizarov, S. (2016). Oil refinery hazardous effluents minimization by membrane filtration: An on-site pilot plant study. J. Environ. Manag., 181, 762-769.

Santos, B., Crespo, J. G., Santos, M. A., and Velizarov, S. (2016). Oil refinery hazardous effluents minimization by membrane filtration: An on-site pilot plant study. J. Environ. Manag., 181, 762-769.

Thibault, Y., Gamage McEvoy, J., Mortazavi, S., Smith, D., and Doiron, A. (2017).

Characterization of fouling processes in ceramic membranes used for the recovery and recycle of oil sands produced water. J. Membr. Sci., 540, 307-320.

Thibault, Y., Gamage McEvoy, J., Mortazavi, S., Smith, D., and Doiron, A. (2017).

Characterization of fouling processes in ceramic membranes used for the recovery and recycle of oil sands produced water. J. Membr. Sci., 540, 307-320.

Weschenfelder S.E., Louvisse A.M.T., Borges C.P., Meabe E., Izquierdo J., and Campos J.C. (2015a). Evaluation of ceramic membranes for oilfield produced water treatment aiming reinjection in offshore units. J. Petrol. Sci. Eng. 131, 51-57.

Weschenfelder S.E., Louvisse A.M.T., Borges C.P., Meabe E., Izquierdo J., and Campos J.C. (2015a). Evaluation of ceramic membranes for oilfield produced water treatment aiming reinjection in offshore units. J. Petrol. Sci. Eng. 131, 51-57.

Weschenfelder, S. E., Borges, C. P., and Campos, J. C. (2015b). Oilfield produced water treatment by ceramic membranes: Bench and pilot scale evaluation. J. Membrane Sci., 495, $242-251$.

Weschenfelder, S. E., Borges, C. P., and Campos, J. C. (2015b). Oilfield produced water treatment by ceramic membranes: Bench and pilot scale evaluation. J. Membrane Sci., 495, 242-251.

Weschenfelder, S. E., Fonseca, M. J. C., Borges, C. P., and Campos, J. C. (2016). Application of ceramic membranes for water management in offshore oil production platforms: Process design economics. Sep. Purif. Technol. 171, 214-220.

Weschenfelder, S. E., Fonseca, M. J. C., Borges, C. P., and Campos, J. C. (2016). Application of ceramic membranes for water management in offshore oil production platforms: Process design economics. Sep. Purif. Technol. 171, 214-220.

Xu, J., Ma, C., Sun, Y., Shi, W., Yu, S., and Wang, L. (2017). Advanced treatment of produced water from asp (Alkali/surfactant/polymer) flooding with polyethersulfone UF membrane for reinjection. Desal. Water Treat., 61, 42-49.

Zhu, X., Dudchenko, A., Gu, X., and Jassby, D. (2017). Surfactant-stabilized oil separation from water using ultrafiltration and nanofiltration. J. Membr. Sci., 529, 159-169.

Zhu, X., Dudchenko, A., Gu, X., and Jassby, D. (2017). Surfactant-stabilized oil separation from water using ultrafiltration and nanofiltration. J. Membr. Sci., 529, 159-169.

Zoubeik, M., Ismail, M., Salama, A., and Henni, A. (2018). New developments in membrane technologies used in the treatment of produced water: A review. Arab. J. Sci. Eng., 43(5), 20932118.

Zoubeik, M., Ismail, M., Salama, A., and Henni, A. (2018). New developments in membrane technologies used in the treatment of produced water: A review. Arab. J. Sci. Eng., 43(5), 2093-2118. 
Zsirai, T., Al-Jaml, A. K., Qiblawey, H., Al-Marri, M., Ahmed, A., Bach, S., Watson, S., and Judd, S. (2016). Ceramic membrane filtration of produced water: Impact of membrane module. Sep. Purif. Technol., 165, 214-221.

Zsirai, T., Al-Jaml, A. K., Qiblawey, H., Al-Marri, M., Ahmed, A., Bach, S., Watson, S., and Judd, S. (2016). Ceramic membrane filtration of produced water: Impact of membrane module. Sep. Purif. Technol., 165, 214-221.

Zsirai, T., Qiblawey, H., Buzatu, P., Al-Marri, M., and Judd, S. J. (2018). Cleaning of ceramic membranes for produced water filtration. J. Petrol. Sci. Eng., 166, 283-289.

Zsirai, T., Qiblawey, H., Buzatu, P., Al-Marri, M., and Judd, S. J. (2018). Cleaning of ceramic membranes for produced water filtration. J. Petrol. Sci. Eng., 166, 283-289. 